\title{
Smart Sentinel: Monitoring and Prevention System in the Smart Cities
}

\author{
J. M. Sánchez Bernabéu, J. V. Berná Martínez, F. Maciá Pérez
}

\begin{abstract}
Today, faced with the constant rise of the Smart cities around the world, there is an exponential increase of the use and deployment of information technologies in the cities. The intensive use of Information Technology (IT) in these ecosystems facilitates and improves the quality of life of citizens, but in these digital communities coexist individuals whose health is affected developing or increasing diseases such as electromagnetic hypersensitivity. In this paper we present a monitoring, detection and prevention system to help this group, through which it is reported the rates of electromagnetic radiation in certain areas, based on the information that the own Smart City gives us. This work provides a perfect platform for the generation of predictive models for detection of future states of risk for humans. Copyright (C) 2014 Praise Worthy Prize S.r.l. - All rights reserved.
\end{abstract}

Keywords: Smart City, Electromagnetic Radiation, Sensor System, Electromagnetic Hypersensitivity, Electro-Sensitivity, Monitoring, Detection, Risk, Internet of Things, Human

\section{Introduction}

Smart Cities appeared by the need to optimize the efforts of the cities in the development of policies that focus on: IT-education, IT-infrastructure, IT-economy and quality of life [1]. The process of incorporating and use in a massive way the IT in the cities, it is called 'smartification' by [2], [3] and the own Institute of Electrical and Electronics Engineers (IEEE) [4], and implies that a city needs a broad deployment of systems and techno-logical devices throughout the city, which can help you transform it and adapt to their sustainability needs to better manage their resources and energy sources [5], [6].

In this process aims to achieve an efficient management of all areas of the city: ur-ban planning, infrastructure, transport, education, health, public safety, energy, natural environment and quality of life by meeting their needs and those of its citizens.

These systems make use of machine to machine infrastructure (M2M) [16], which al-lows the integration in the platform of devices defined in a Smart City, connecting via gateways $\mathrm{M} 2 \mathrm{M}$ or through the interconnection between heterogeneous devices.

Nevertheless, the technology used is capable of generating a complex combination of weak electric and magnetic fields, also called electro pollution, that normally do not affect the majority of the population but there is a collective of people, according to studies by [7], [8], [17], which is affected by this exposure studied by [18]-[20], presenting symptoms such as headaches, memory loss, sleep disorder, blurred vision, nausea, or fatigue.
It has been suggested that exposure to magnetic fields at power frequencies $(50 / 60 \mathrm{~Hz})$, that is to say, extremely low frequency (ELF), could lead to an increased incidence of cancer in children and other adverse health effects. The evidence comes mainly from epidemiological studies in residential areas. These studies suggest that there is a partnership between children's exposure to ELF magnetic fields and the increased risk of leukemia [9]. Electromagnetic fields (EMF) are characterized by its wavelength or frequency in a radio-active or two categories (Table I illustrates the features of each one of them):

- Non-ionizing: low level of radiation which generally sees the human being but without causing serious injuries.

- Ionizing: can alter the DNA due to their potency.

TABLE I

Classification Of Electromagnetic Fields (SOURCE [10])

\begin{tabular}{ccc}
\hline \hline Definition & Forms of radiation & Examples \\
\hline $\begin{array}{c}\text { Non-Ionizing } \\
\text { Low to mid frequency, } \\
\text { which is generally }\end{array}$ & Extremely Low & Extremely Low \\
perceived as harmless & Frequency(RF) & Radio Frequency(RF) \\
due to its lack of & Microwaves & Microwaves \\
potency & Visual Light & Visual Light \\
Ionizing & & \\
Mid to high frequency & & Ultraviolet Light \\
radiation which can, & & X-ray range between \\
under certain & Ultraviolet(UV) & $30 * 1016 * 30 \mathrm{~Hz}$ to \\
circumstances, lead to & X-Rays & $1019 \mathrm{~Hz}$ \\
cellular and DNA & Gamma & Some Gamma Rays \\
damage with & & \\
prolonged exposure & &
\end{tabular}


The concept of Smart City allows us to have access to a large volume of data generated by the various types of technological resources in real time, processing them, and developing new analytical tools for a great value to help this group of persons hypersensitive.

To meet the new challenges in the Smart Cities arises the need for control panels or applications, through which users or citizens, with some type of hypersensitivity to this type of radiation, to have information in real time and deferred of the levels of EMF as the zone where people move, to try to avoid its prolonged exposure. There are proposals in which deals with the measurement of EMF for monitoring such as those of Urbinello [11] or Huss [12], but suffer from the fact that require the installation of new measurement devices or Smart Meters that further enhance the magnitude of the EMF radiation.

In order to give a solution to this problem, this work provides a monitoring and in-formation system which allow us to measure the electromagnetic radiation level in buildings and cities, allowing its use in both real-time and deferred.

This system will allow you to use a smartphone by way of such tool that allows us to define an alert level to inform users of the proximity of the antennas and the levels of radiation that can generate, advising as well to the users of the possibility of being affected by this radiation, this tool will serve as a basis for future lines of development which may be incorporated into the tool to other risk factors based on the monitored data and even the generation of predictive models based on the existing historical data.

\section{Proposal: Smart System Sentinel EMF}

The system that we present what we have called Smart Sentinel: monitoring of risks system in Smart Cities, and in particular we are going to focus on the Non-ionizing EMF fields generated by the different electrical and electronic devices existing anywhere.

We propose a system for process, analyze, monitor and generate scorecards in real time and deferred, in which the user may display the radiation levels caused by the non-ionizing EMF fields in any area where there is a smart infrastructure. With this approach the users will be able to avoid prolonged exposure in certain areas, as to day of today do not have that knowledge, and alleviate the symptoms produced by electromagnetic hypersensitivity. To achieve the objective of our proposal we relied on Smart City generic architectural view defined by levels as proposed in [9].

Smart Sentinel System acts as a layer to cross all the levels defined in a Smart City architecture (see Figure 1 and Figure 2). This layer is fed by all the levels defined in it, from the level of monitoring in which you specify all the sensors and electronic devices and infrastructure, the level of business where we will store and we will process the data obtained in the previous level, the level of implementation where we will generate value-added resources such as web applications, standalone and mobile apps, and the level of communications which will allow you to get to know the infrastructure of internetworking, our aim is to be able to have all the necessary information about the technical characteristics of all the devices, the data recorded by each of them, the data and use of radiation emitted at each moment and the geographical positions, in order to process and analyze the information and offer services to users and systems or devices that can consume the type of information that we provide.

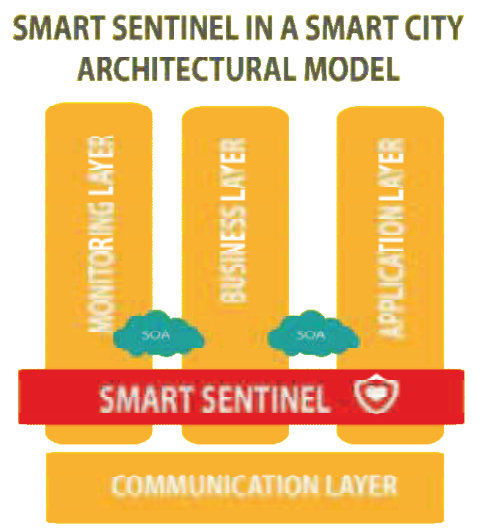

Fig. 1. Smart Sentinel on Smart City architecture

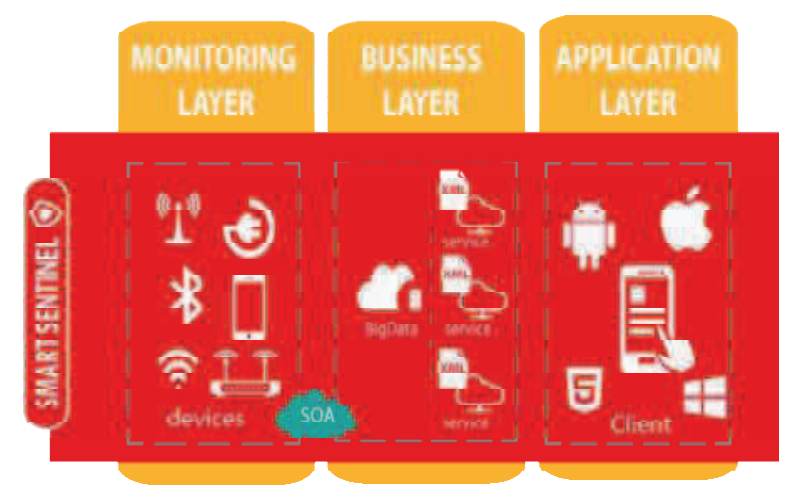

Fig. 2. Smart Sentinel internal level on Smart City architecture

At the internal level Smart Sentinel System makes use of all information generated by all levels of the Smart City architecture making intensive use of the level of business, this level will be responsible for generating the information in web services.

\section{II.1. Monitoring Level}

In this level we use sensors, wireless systems, internetworking devices and electrical networks, which receive and transmit information and energy respectively for the environment in which have been integrated.

The following devices are included in the list: smartphones, handhelds, and tablets that are connected via wifi and make use of the network.

These two sets of devices provide us the information by which we are going to perform the implementation of 
our system.

The wireless systems employees are able to provide real time information about connections made and the level of data issued by each one of them, have a Serviceoriented architecture (SOA) Services that feed information to business level for the subsequent analysis of the data proposed by [15].

By the electrical networks we know information on the purchases made in real time, the infrastructure deployed throughout the area and the power handling. As we have explained in section 1, which describes the state of the art, these technologies are basically $\mathrm{M} 2 \mathrm{M}$ and services are available to interact with the business layer.

As we have said before in the preceding section, we don't employ an external measurement devices such as Smart Meters, already they would add a higher rate of radiation in the Smart City and therefore harmful to the user.

\section{II.2. Business Level}

This level is responsible for collecting, standardizing, process, store, analyze isolated data and massive and transform them into useful information in addition under the paradigm of service, and provide services that provide interoperability, integration, expandability and compatibility between layers.

A part of the data provided by sensors, wireless devices, and internetworking devices, this layer will have other detailed information such as: the technical sheets of all types of devices (density of emitted power, range expansion of the signal, channels, gain, sensitivity of the receptors, operating temperature, among others) and historical information about power consumed.

With all this information and storage systems and analysis can be employed as relational database management system (RDBMS), Data Mining, Data Warehouse, Big Data, we can generate information and offer it through the SOA paradigm to the clients or devices who want to make use of it.

For the distribution of data and services and applications, you can use the cloud as a common platform for data management and distribution as proposed by Armbrust in [13] or Buyya in [14].

\section{II.3. Application Level}

Through the development of applications for all types of devices such as smartphones, tablet, and desktop, the user will be able to dispose of information in real-time, historical or deferred about electromagnetic radiation and the areas with the greatest influx according to the need for it, because that will be able to make use of historical data for the generation of more reliable statistics and build predictive models.

The application layer will provide information about the status of a given area and, through a system of reminders, will offer the information to user if you are coming to the spaces with the highest rate of radiation.
This application can work in online mode, but also in offline mode, for those users who are unable to make use of the data connection at all times because of their hypersensitivity, down-loading all the data needed to display the maps EMF.

This layer combines information from different sources to provide a greater level of detail, such as:

- Network Connections of networking devices

- Sheet of technical characteristics of each device

- Network Connections for smartphones, tablets and pc

- Electrical Connections in different locations

- Historical connections and electrical consumption

- Drivers of consumption of the devices themselves

For the distribution of data and services and applications, you can use the cloud as a common platform for data management and distribution.

\section{II.4. Communications Level}

As mentioned above, the support of this level to our proposal is crucial, as it will sup-port services transactions that are generated by the services layer. At this level makes use of interconnect systems equipment and internetworking.

\section{Prototype}

In order to test our system we have developed an application for Android devices. This application makes use of the service in real-time geolocation of the mobile de-vice, or in offline mode if you want to, and the Google API to provide information about the index of radiation to which you are subject, both inside and outside of the area.

In this case a simulation has been made in the facilities of the General Library of the University of Alicante, which has a large amount of wireless devices, servers, routers, and electrical panels in certain areas of the same.

\section{III.1. Characteristics of the information}

To provide actual data to users, we have used the information provided by each of the devices on which we rely to generate our maps of EMF radiation in our prototype. We can distinguish two different types of sources of information:

Data streaming

- Wireless access points: for these types of devices we get existing connections in every instant in the wireless nodes, MAC, IP, or the name of the connected device. These data are obtained by the level of business and, after its convenient processing, services are offered through the level of application

- Processing devices: the services of business level are responsible for monitoring devices that are active on our system, such as routers, application servers, storage servers, information panels and in general all those devices that are part of the infrastructure to 
provide an estimate of areas with EMF radiation due to consumption of these devices.

Stored Data

- Technical Information: in the level of business will incorporate the technical information on the infrastructure and devices that are part of the facilities to be monitored, as can be routers, antennas, electrical panels, lighting, electrical lines, network wiring and in general any other electronic device.

- Consumption data: by the providers of services are obtained information concerning the global electrical consumption, peaks of electrical consumption, consumption by building and consumption of telecommunications network.

It should be noted that the devices connected to the data network can be monitored from the level of business, at least to know if it is active or not. This we know, for example, how many of the computers inventoried in the field of control (for ex-ample the University of Alicante) are consuming resources and issuing EMF radiation

\section{III.2. Viewing the Information}

The application is composed of two display modes of the commented previously information. A spatial mode in which we can combine all the information on the different types of data to give a vision to the user of the space that surrounds him and the EMF influence in your environment, and other display in meter mode that provides a simplified view of the EMF level of the current geographic location.

In the spatial mode of display we inform the user of the areas of radiation in the location in which it is situated. Shown here is a map, using the Google API, which highlights the user's current location and placed various elements that produce influence EMF in addition to various levels. One of the most important elements are the nodes of wireless access, in Fig. 3 we can see the representation of the wireless access points around the user that is currently in the general library of the UA campus.

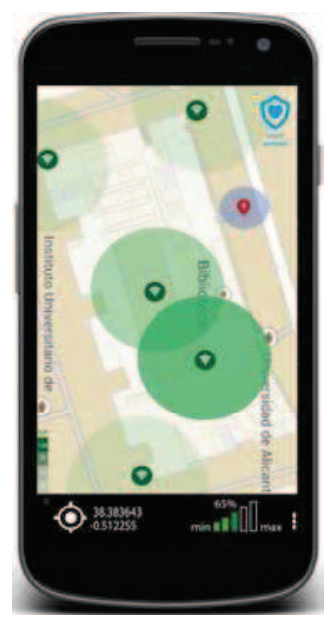

Fig. 3. Spatial Mode
Each of these nodes is represented by an area of influence that takes a color more or less intense depending on the signal from the antenna, the number of Wi-Fi connections that are enduring that node and the activity of the device itself.

On this map are formed by juxtaposing the different elements that produce the risk that we are being monitored, in addition to nodes of Wi-Fi access, also shows the electrical devices, areas of transformers, electrical consumption of a building, and the processing devices such as routers and servers. In Fig. 4(a) we can see a complete representation of all the elements of influence EMF that the application collects, along with the current position of the user, in this way through the greater or lesser intensity of the color that represents the EMF radiation a user can have an idea of which areas are potentially dangerous for and therefore should be avoided, also this figure represents the streaming mode of the application, which displays the data in real time.

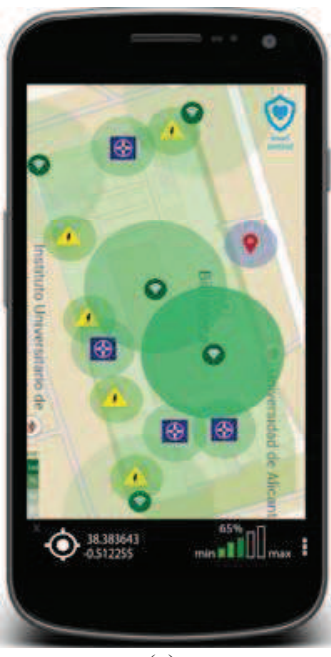

(a)

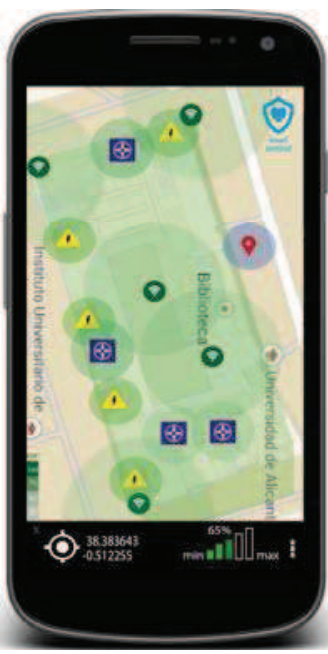

(b)
Figs. 4. (a) Streaming Mode (b) Offline Mode

In Fig. 5 we can visualize the configuration screen of the application, which allows us to mark items we want to represent on the map.

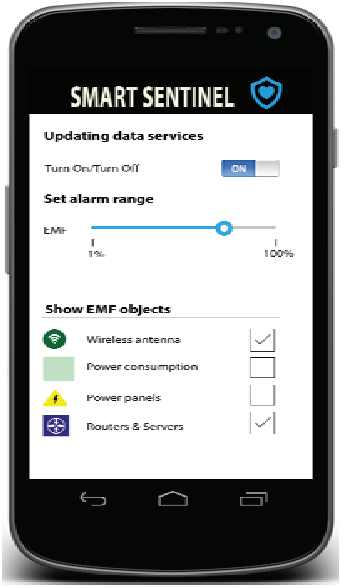

Fig. 5. Setup Mode 
The application enables us to operate in offline mode or deferred (see Fig. 4(b)). This mode does not perform any data update and does not receive data from the streaming services, i.e. does not display data of incidence EMF in real time.

When the application is in this mode, the map does not update the elements whose information depend on this service of streaming data, such as the signal strength of the wireless access nodes, or just stop render if we have no information about them. Only shows the data received in your last connection and static data tab as technical and historical data of the devices that emit EMF. In the lower left area of the screen we can see the map legend of EMF radiation, which has a scale of colors ranging from the 1 , minor radiation index, to 100 , area of greatest impact (see Fig. 6(a)).

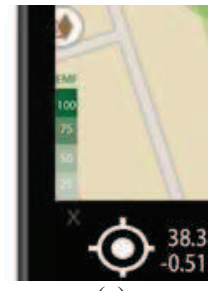

(a)

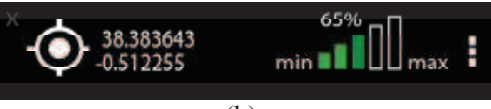

(b)
Figs. 6. (a) Legend and (b) radiation index scale

In the lower area of the screen we can see the position of the user in coordinates along with the value of EMF radiation in that position, which has a scale of values ranging from 1 ( $\mathrm{min})$, less radiation index, to 100 (max), area of greatest impact (see Fig. 6(b)).

In Meter mode the application Smart Sentinel will allow us to inform the user of the index of EMF radiation in its current location.

It has an alert mode, see left side of Fig. 7(a), which prompts you through a red button (see Fig. 7(b)), if you have exceeded the limits indicated in the configuration of smart alarms Sentinel.

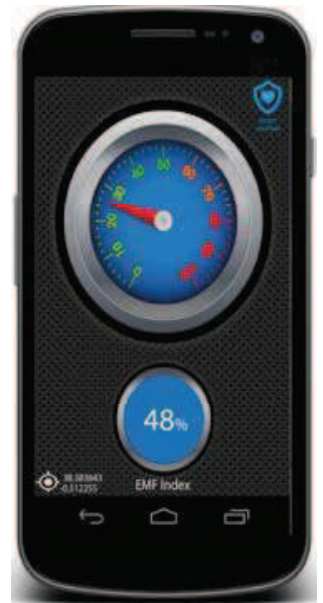

(a)

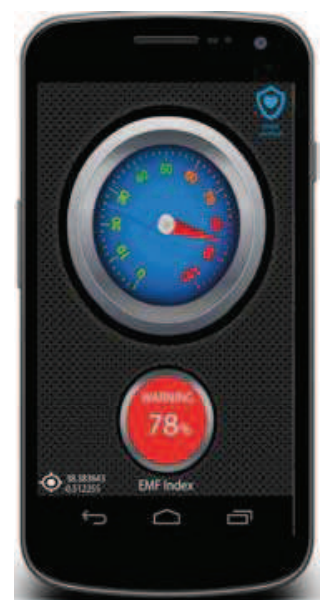

(b)
Figs. 7. (a) Meter Mode without alarm.

(b) Meter Mode with alarm
The alert level is customizable via the configuration screen that has been seen in Fig. 8, thereby the user to set the maximum level of radiation in which he wants to be contacted depending on your tolerance.

In addition to the two display modes shown, there is also an area of customization and configuration as we have seen in the Fig. 5, and information display (Fig. 8) where are identified the items represented in the application.

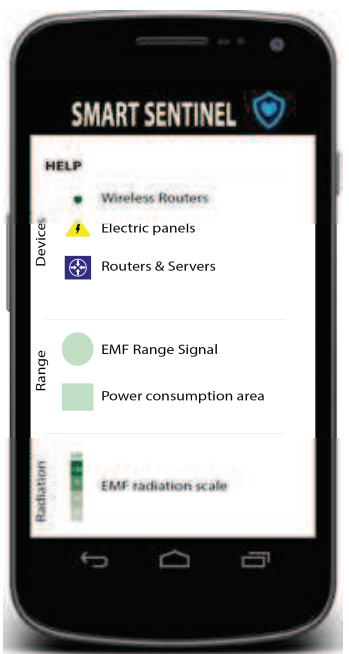

Fig. 8. Help Screen

\section{Conclusions and Future Work}

In this paper, a proposal has been presented for the development of a monitoring and information, in realtime or delayed, of the index of electromagnetic radiation of buildings and areas of a Smart City, embodied for our university, and dedicated to users who suffer the pathology of electromagnetic hypersensitivity.

The approach has allowed us to define a prototype capable of taking advantage of existing infrastructures and the historical and technical data of the Smart Cities, without adding new elements such as the Smart Meterings, which increases the level of existing radiation. The proposed system has advantages over the actual monitoring system because our system don't require the installation of new measurement devices. We seek to do so through the information supplied by the own devices that form the infrastructure, covering the development under the architectural model of Smart City, in which the initiatives it are built in an integrated manner. Our system makes use of all information provided by the various levels as the basis for the Smart City, and therefore is able to integrate in the proposal any other sensing element or data that you have accommodated within the paradigm Smart City.

In the short term we are considering incorporating in the monitoring system of other risk factors such as could be pollution, pollen, noise or environmental factors. To do this in the proposal it would suffice to integrate other sensing devices and data services to reflect on our map the various risk factors in addition to the EMF. 
In the medium term, using all the information stored and with enough baggage, we could generate prediction models (for example as do meteorological models), to facilitate not only monitoring but also being able to predict the occurrence of these risk factors in the areas of interest to users, thus generating preventive tools.

\section{Acknowledgements}

This work has been performed within project Smart University funded from the Vice President office for Information Technology at the University of Alicante.

\section{References}

[1] A. Mahizhnan, Smart Cities: The Singapore Case, Cities, Vol. 16, n. 1, pp. 13-18, 1999

[2] S. Bartolini, B. Milosevic, A. D'Elia, E. Farella, L. Benini, T. S. Cinotti, Natural interaction in Reconfigurable smart environments: Approach and prototype implementation, Personal and Ubiquitous Computing. Vol. 16, n. 7, pp. 943-956, 2012.

[3] S. Ruiz-Romero, A. Colmenar-Santos, F. Mur-Pérez, A. LópezRey, Integration of distributed generation in the power distribution network: The need for smart grid control systems, communication and equipment for a smart city, Renewable and Sustainable Energy Reviews, Vol. 38, pp. 223-234, 2014.

[4] IEEE Smart Cities Iniciative [Online], 2014. http://smartcities.ieee.org/home/ieee-invites-globalmunicipalities-to-engage-in-new-ieee-smart-cities-initiative.html

[5] J. P. Vasseur, A. Dunkels, in Jean-Philippe Vasseur and Adam Dunkels, Morgan Kaufmann (ed.), Smart Cities and Urban Networks, In Smart Objects with Interconnecting IP, (Boston 2010, 335-251)

[6] D. Urbinello, J. Wout, A. Huss, L. Verloockc, J. Beekhuizend, R.Vermeulend, L. Martens, M. Röösli, Radio-frequency electromagnetic field (RF-EMF) exposure levels in different European outdoor urban environments in comparison with regulatory limits, Environment International, Vol. 68, pp. 49-54, 2014.

[7] M. Havas, Electromagnetic hypersensitivity: biological effects of dirty electricity with emphasis on diabetes and multiple sclerosis, Electromagn Biol Med, Vol. 25, n. 4, pp. 259-268, 2006.

[8] A. Caragliu, C. Del Bo, P.Nijkamp, Smarts cities in Europe, Journal of urban technology, Vol. 18, n. 2, pp. 65-82, 2011.

[9] I. Calvente, M.F. Fernandez, J. Villalba, N. Olea, M.I. Núñez, Exposure to electromagnetic fields (non-ionizing radiation) and its relationship with childhood leukemia: A systematic review. Science of the total environment Vol. 408, Issue 16, 15, pp. 30623069, 2010.

[10] National Intitute of Environmental Health Sciences [online] 2014http://www.niehs.nih.gov/health/topics/agents/emf/

[11] D. Urbinello, A. Huss, J. Beekhuizen, R. Vermeulen, M. Röösli, Use of portable exposure meters for comparing mobile phone base station radiation in different types of areas in the cities of Basel and Amsterdam. Science of Total Environment, Vol. Jan 15, pp. 468-469:1028-1033, 2014

[12] A. Huss, D. Urbinello, W. Joseph, L. Verloock, J. Beekhuizen, R. Vermeulen, L. Martens and M. Röösli, Radio-frequency electromagnetic field (RF-EMF) exposure levels in different European outdoor urban environments in comparison with regulatory limits. Environment International. Vol. 68, pp. 49-54, 2014.

[13] M. Armbrust, A. Fox, R. Griffith, , A.D. Joseph, R. Katz, A. Konwinski, G. Lee, D. Patterson, A. Rabkin, I. Stoica, and M. Zaharia,. A view of cloud computing. Communications of the ACM.Vol. 53, N.4, pp. 50-58, 2010.

[14] R. Buyya, J. Broberg and A. Goscinski. Cloud Computing: Principles and Paradigms. (John Wiley \& Sons), 2011

[15] K. Barry, D. Dick, D. Managing Change with Incremental SOA Analysis, In The Savvy Manager Guide (Ed). Web Services,
Service-oriented Architectures, and Cloud Computing (Second Edition). (Boston, Morgan Kaufmann, 2013, 113-127.

[16] A. Elmangoush, H. Coskun, S. Wahle, T. Magedanz. Design aspects for a reference M2M communication platform for Smart Cities. Proceedings of the 9th International Conference on Innovations in Information Technology, IIT 2013(Page: 204-209, Year of Publication: 2013. ISBN: 978-146736203-0).

[17] A. Ahlbom., U. Bergqvist, J.H. Bernhardt, J.P. Cesarini. Guidelines for limiting exposure to time-varying electric, magnetic, and electromagnetic fields (up to $300 \mathrm{GHz}$ ). Health Physics. Vol. 74, n. 4, pp. 494-521, 1998.

[18] A. Bürgi, G. Theis, A. Siegenthaler, M. Röösli. Exposure modeling of high-frequency electromagnetic fields. Journal of Exposure Science and Environmental Epidemiology. Vol. 18, n. 2, pp. 183-191, 2008.

[19] D. Urbinello, M. Röösli. Impact of one's own mobile phone in stand-by mode on personal radiofrequency electromagnetic field exposure. Journal of Exposure Science and Environmental Epidemiology. Vol. 23, n. 5, pp. 545-548, 2013.

[20] M. Khalid, T. Mee, A. Peyman, D. Addison, M. Maslanyj, S. Mann. Exposure to radio frequency electromagnetic fields from wireless computer networks: Duty factors of Wi-Fi devices operating in schools. Progress in Biophysics and Molecular Biology. Vol. 107, n. 3, pp. 412-420, 2011.

\section{Authors' information}

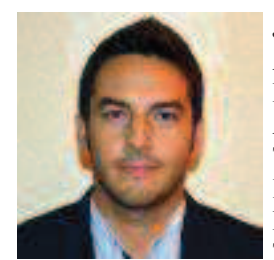

J. M. Sanchez Bernabeu $\mathrm{He}$ was born in Alicante (Spain). He received the Bachelor's Degree in Computer Science from University of Alicante in 2010 and Master in Computer Technologies in 2014. Now is student PhD in Information Technologies. And is part of Middleware Group in Department of Computer Technology in University of Alicante. He's working with M2M Communications, Smart Cities and Internet of the Things.

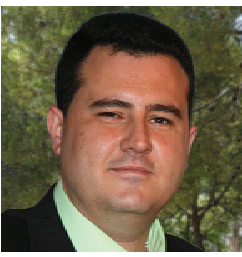

Jose Vicente Berna-Martinez was born in Spain in 1978. He received his engineering degree and the $\mathrm{Ph} . \mathrm{D}$. degree in Computer Science from the University of Alicante in 2004 and 2011 respectively. From 2006 to 2013, he was an Associate Professor at the University of Alicante, currently he is a Assistant doctor. His research interests are in the area of computer networks, distributed systems, bio-inspired systems and robotics which are applied to industrial problems.

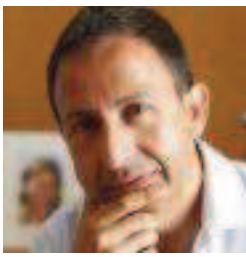

Francisco Maciá-Pérez was born in Spain in 1968. He received his engineering degree and the Ph.D. degree in Computer Science from the University of Alicante in 1994 and 2001 respectively. He worked as System's Administrator at the University of Alicante form 1996 to 2001. He was an Associate Professor from 1997 to 2001. Since 2001, he is a Professor and currently he is the Vice President for Information Technologies at the University of Alicante. His research interests are in the area of network management, computer networks, smart sensor networks and distributed systems, which are applied to industrial problems. 